Article

\title{
Double-Sided Freeform Lens for Light Collimation of Light Emitting Diodes
}

\author{
Yong-Sin Syu, Chun-Ying $\mathrm{Wu}(\mathbb{D}$ and Yung-Chun Lee * \\ Department of Mechanical Engineering, Cheng Kung University, Tainan 701, Taiwan; \\ mg5510633@gmail.com (Y.-S.S.); chunying1009@gmail.com (C.-Y.W.) \\ * Correspondence: yunglee@mail.ncku.edu.tw; Fax: +886-6-2094-010
}

Received: 23 October 2019; Accepted: 9 December 2019; Published: 12 December 2019

check for updates

\begin{abstract}
A double-sided freeform lens is proposed for collimating light emitted from light emitting diodes (LEDs). The surface profiles of the lens are mathematically characterized and precisely determined based on a point-source assumption and differential geometry theory. The proposed lens design method is straightforward, flexible, and effective. Moreover, the optical performance of the lens can be intuitively adjusted by tuning just a small number of design parameters. The simulation results showed that the proposed lens achieved an excellent collimating effect for a commercial ultraviolet (UV) LED. A prototype lens is fabricated in UV-grade poly(methyl methacrylate) material using a standard injection molding process. The light collimating effect of the lens/UV-LED assembly was measured experimentally and was shown to be in good agreement with the simulation results. The collimating angle at the half-energy level was equal to $1.88^{\circ}$. The performance of the UV-LED is thus comparable to that of conventional lithography UV light sources based on mercury arc lamps. Consequently, the proposed double freeform lens showed significant potential for photolithography applications within the industry.
\end{abstract}

Keywords: lenses; light emitting diodes; ultraviolet light; differential geometry; geometrical optics; machining; lithography; collimator surfaces

\section{Introduction}

Compared to the traditional UV mercury lamps used in conventional photolithography systems, ultraviolet (UV) light emitting diodes (LEDs) have the advantage of having a lower cost, a reduced electricity consumption, a longer lifetime, improved stability, and being more environmentally friendly. As a result, there has been growing interest in it being a potential light source for UV lithography processes. However, collimating the UV light without losing too much of the light energy represents a major challenge and requires the use of special lens designs.

Both primary and secondary optics are commonly adopted to adjust the distribution of light so as to meet the overall specifications and create the desired beam pattern. Freeform surfaces, in particular, have been widely used to refract and/or reflect LED light. For example, the authors in [1-3] used lenses with a concave spherical surface on the LED side and a freeform refractive surface on the other side to achieve a uniform illumination intensity over a rectangular area. In contrast, the authors in [4-6] used double freeform surfaces to improve the optical performance by refracting the LED light twice. In many cases, the freeform lenses are intimately integrated with the LED packaging in order to achieve compactness [7,8], or better angular color uniformity [9]. For LEDs with a wide radiation angle, more complex freeform surfaces are commonly employed to refract the light at a lower angle and reflect the light at a higher angle [10-17]. The surface profiles in such lenses typically comprise of several discontinuities separating a small number of analog profile sections with different functions. The resulting freeform surfaces are generally designed using numerical schemes and 
computer algorithms. However, Fresnel surfaces have also been proposed for redistributing LED light on the reflection surface $[18,19]$. Proposed freeform lens share the same purpose of many other lens designs, such as the positive meniscus lens [20], by minimizing the spherical aberration. However, conventional positive meniscus lenses consist of two spherical lens surfaces, and hence their light collimating capability is limited only to paraxial rays. In practice, most LEDs have a large radiation angle. Therefore, they cannot be easily collimated to a small collimating angle by a conventional meniscus lens without losing the majority of their light energy. Furthermore, most commercial positive meniscus lenses have a relatively long focal length of around 100 to $1000 \mathrm{~mm}$ and are therefore not easily integrated with LEDs.

The majority of previous studies on the use of freeform surfaces for LEDs consider the problem of optical lighting, which generally requires a certain distribution of illumination intensity on a target area that is placed quite far from the LED. In such situations, the light collimating effect is not a significant concern, provided that the light beam has a limited viewing angle in the far field. As a result, when designing the lens, emphasis is placed mainly on the illumination uniformity and light efficiency. However, when applying UV-LEDs to photolithography applications, light collimation is a major concern since it has a direct effect on the dimensional accuracy of the developed photoresist (PR) microstructure. Furthermore, in photolithography, the UV light source is relatively close to the targeted PR layer, and hence the UV light should be collimated immediately after the freeform lens in the near field. However, the uniformity of the projected light intensity is less important than in general optical lighting applications since the UV patterned area is typically in the wafer size (up to $12^{\prime \prime}$ ) or panel size (up to $28^{\prime \prime}$ ) and hence illumination is generally achieved using an array of lens/LED units in which the overlapping exposures of the multiple units are controlled using a programmed scanning movement [16].

The present study proposes a single lens with two freeform lens surfaces to collimate the light emitted from an UV-LED. The two surfaces are characterized mathematically as functions in a polar coordinate system. Furthermore, based on fundamental ray optics and differential geometry, the solutions for the two lens surfaces are analytically determined using a point source assumption and an imaginary point source. One of the freeform lens profiles is solved numerically from a simple ordinary differential equation, while the other has a closed-form solution. The designed double freeform lens is evaluated both numerically and experimentally for a real UV-LED. It is shown that the light beam emitted from the UV-LED is refracted twice by the designed freeform lens' surface and is incident on the target image plane with a good collimation accuracy.

\section{Double Freeform Lens Design for Collimating Light from Point Source}

This paper proposes a method for designing a LED light collimating lens with two analog freeform surfaces. As shown in Figure 1a, the LED light source was first approximated as a point source, $S$, located at the origin of a $(x, z)$ coordinate system in which the $+z$-axis is the optical axis of the LED. The lens under consideration was axially symmetrical with respect to the $z$-axis. Therefore, the $x$-axis could be any direction perpendicular to the $z$-axis. It is noted that a real LED is not a point source and its radiation pattern may thus deviate slightly from axial-symmetry. However, after completing the lens design, a real LED light source was modeled with the designed freeform lens and the overall optical performance was evaluated. In addition, the designed lens was fabricated and characterized experimentally using a commercial UV-LED.

As shown in Figure 1a, the two freeform lens surfaces are denoted as $S_{1}$ and $S_{2}$, respectively. The lens was designed such that the light emitted from the point source $S$ was refracted at points $P$ and $P^{\prime}$ by surfaces $S_{1}$ and $S_{2}$, respectively, and became parallel to the $z$-axis in air as a result. The optical refractive index of the lens material is $n$. The key element in the proposed freeform lens design method was to individually assign surfaces $S_{1}$ and $S_{2}$ with different functions such that they perfectly collimate the light emitted from the point source, $S$. The function of surface $S_{1}$ was to refract all rays emitted by $S$ in such a way that they appear to originate from a new imaginary point source $S^{\prime}$ (see Figure 1a,b). 
Let $r_{o}$ be the distance between the point source $S$ and the apex point $A$ of surface $S_{1}$. Note that the location of $S^{\prime}$ is chosen to be at $z=-\xi r_{0}$ where $\xi$ is a parameter to be determined. As shown in Figure 1c, the function of surface $S_{2}$ is to refract all of the rays emitted from point $S^{\prime}$ at the lens/air interface such that they become parallel to the $+z$ axis in air.
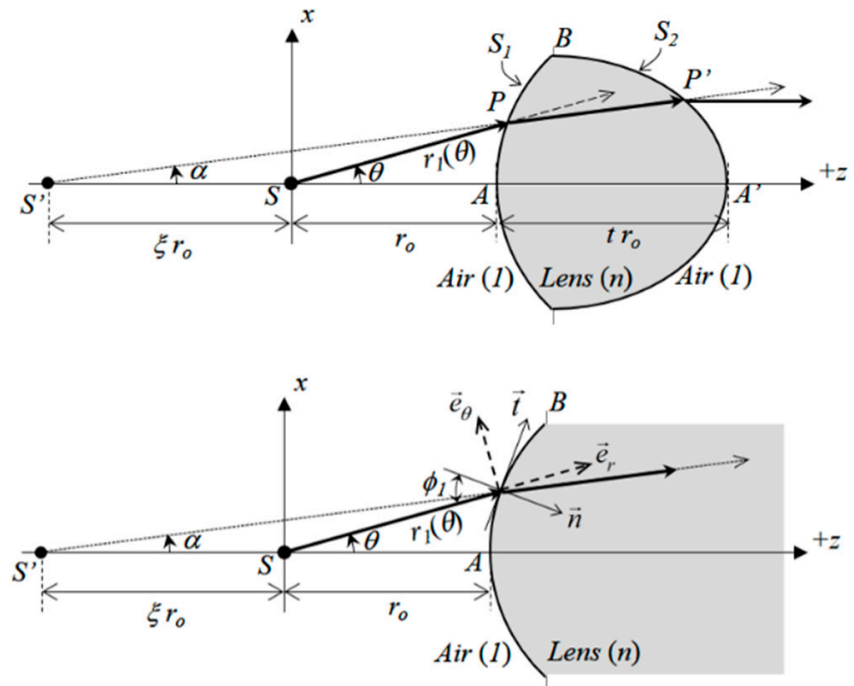

(b)

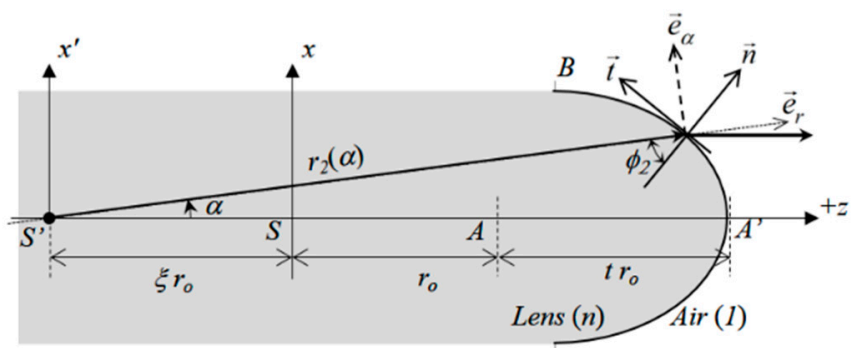

Figure 1. Double freeform lens for collimating light from point source: (a) Overview, (b), and (c) details for constructing front $\left(S_{1}\right)$ and back $\left(S_{2}\right)$ surfaces of lens.

To mathematically derive the equations and solutions for surfaces $S_{1}$ and $S_{2}$, the present analysis adopted the polar coordinate systems $\left(r_{1}, \theta\right)$ and $\left(r_{2}, \alpha\right)$ shown in Figure $1 \mathrm{~b}, \mathrm{c}$, respectively. The lens design problem then becomes one of finding the functions of $r_{1}=r_{1}(\theta)$ and $r_{2}=r_{2}(\alpha)$ which uniquely characterize and represent surfaces $S_{1}$ and $S_{2}$, respectively, and fulfill the refractive functions assigned to them.

As shown in Figure 1b, let $\vec{n}$ and $\vec{t}$ be the normal and tangential unit vectors of surface $S_{1}$ at an arbitrary point $P$, respectively, and let $\vec{e}_{r}$ and $\vec{e}_{\theta}$ be the two unit base vectors of polar coordinate $\left(r_{1}, \theta\right)$. Furthermore, let the angle between the incident ray and the normal direction $\vec{n}$ be denoted as $\phi_{1}$. From simple geometry and Snell's law, it follows that:

$$
r_{1} \sin \theta=\left(\xi r_{0}+r_{1} \cos \theta\right) \tan \alpha
$$

and

$$
1 \cdot \sin \phi_{1}=n \cdot \sin \left[\phi_{1}-(\theta-\alpha)\right]
$$

Since $\vec{r}_{1}=r_{1} \vec{e}_{r}, \frac{d \vec{r}_{1}}{d \theta}=\frac{d r_{1}}{d \theta} \vec{e}_{r}+r_{1} \vec{e}_{\theta}$, and $\vec{t}$ is parallel to $\frac{d \vec{r}_{1}}{d \theta}$, one has

$$
\tan \phi_{1}=\frac{1}{r_{1}}\left(d r_{1} / d \theta\right)
$$


From Equation (2), one can derive:

$$
\tan \phi_{1}=\frac{\sin (\theta-\alpha)}{\cos (\theta-\alpha)-(1 / n)}
$$

Furthermore, from Equation (3), Equations (4) and (1), one has:

$$
\frac{d r_{1}}{d \theta}=\left[\frac{\sin (\theta-\alpha)}{\cos (\theta-\alpha)-(1 / n)}\right] \cdot r, \text { and } \alpha=\tan ^{-1}\left(\frac{r_{1} \cdot \sin \theta}{\xi \cdot r_{o}+r_{1} \cos \theta}\right)
$$

Equation (5) can be viewed as a first order ordinary differential equation (ODE) for function $r_{1}(\theta)$, which mathematically characterized freeform surface $S_{1}$. Due to its complexity, the ODE cannot be solved analytically. However, the solution of $r_{1}(\theta)$ can be precisely calculated by converting Equation (5) into an equivalent finite difference equation, i.e.,

$$
\begin{gathered}
r_{1}\left(\theta_{i+1}\right)=r_{1}\left(\theta_{i}\right)+\left[\frac{\sin \left(\theta_{i}-\alpha_{i}\right)}{\cos \left(\theta_{i}-\alpha_{i}\right)-(1 / n)}\right] \cdot r_{1}\left(\theta_{i}\right) \cdot\left(\theta_{i+1}-\theta_{i}\right) \quad i=0,1,2, \ldots \\
\alpha_{i}=\tan ^{-1}\left(\frac{r_{1}\left(\theta_{i}\right) \cdot \sin \theta_{i}}{\xi \cdot r_{o}+r_{1}\left(\theta_{i}\right) \cdot \cos \theta_{i}}\right) \quad i=0,1,2, \ldots
\end{gathered}
$$

along with a given initial condition:

$$
r_{1}\left(\theta=0^{\circ}\right)=r_{0}
$$

Based on the explicit numerical marching scheme shown in Equation $(6 \mathrm{a}, \mathrm{b})$, the discrete data of $r_{1}(\theta)$ at different angles can be calculated precisely using a very small angular increment. The calculation of $r_{1}(\theta)$, or the surface of $S_{1}$, can proceed to any arbitrary stopping point $B$, as shown in Figure $1 \mathrm{~b}$. Let $\theta_{B}$ be the angle of point $B$ with respect to the $+z$ axis when viewed from point source $S$. In other words, $\theta_{B}$ is the light collecting angle of the designed lens from the point source.

A similar approach can be taken for analyzing surface $S_{2}$ under the polar coordinate system $\left(r_{2}, \alpha\right)$. As shown in Figure 1c, the polar coordinate system was mounted at the imaginary point source $S^{\prime}$ with two unit base vectors, $\vec{e}_{r}$ and $\vec{e}_{\alpha}$, respectively. $\vec{n}$ and $\vec{t}$ again denote the normal and tangential unit vectors of surface $S_{2}$ at the refraction point $P^{\prime}$, respectively. Furthermore, $\phi_{2}$ denotes the incident angle. After refraction, the ray should be parallel to the $+z$ axis. Thus, based on simple geometry and Snell's law, it follows that:

$$
n \sin \phi_{2}=1 \cdot \sin \left(\phi_{2}+\alpha\right)
$$

or

$$
\tan \phi_{2}=\frac{\sin \alpha}{n-\cos \alpha}
$$

Since $\vec{r}_{2}=r_{2} \vec{e}_{r}, \frac{d \vec{r}_{2}}{d \alpha}=\frac{d r_{2}}{d \alpha} \vec{e}_{r}+r_{2} \vec{e}_{\alpha}$, and $\vec{t}$ is parallel to $\frac{d \vec{r}_{2}}{d \alpha}$, one has:

$$
\tan \phi_{2}=-\frac{1}{r_{2}}\left(d r_{2} / d \alpha\right)
$$

Furthermore, from Equations (8) and (9) one has:

$$
\frac{1}{r_{2}} d r_{2}=\frac{-\sin \alpha}{n-\cos \alpha} d \alpha
$$

Integrating both sides, the analytical solution for $r_{2}(\alpha)$ becomes:

$$
r_{2}(\alpha)=r_{2 *} \cdot\left(\frac{n-\cos \alpha_{*}}{n-\cos \alpha}\right)
$$


where $\left(r_{2 *}, \alpha_{*}\right)$ are integration constants and can be the coordinates of any arbitrarily chosen point on the curved surface $S_{2}$. One obvious choice is point $B$, i.e., the ending point of $S_{1}$ since, by choosing this point, one can connect profiles $S_{1}$ and $S_{2}$ at point $B$, as shown in Figure 1 . Let $\left(r_{2 B}, \alpha_{B}\right)$ be the coordinates of point $B$ in the coordinate system $\left(r_{2}, \alpha\right)$. The lens profile of $S_{2}$ can then be analytically determined in the following mathematically closed-form:

$$
r_{2}(\alpha)=r_{2 B} \cdot\left(\frac{n-\cos \alpha_{B}}{n-\cos \alpha}\right)
$$

The apex point $A^{\prime}$ of surface $S_{2}$, as indicated in Figure 1 , can be determined by setting $\alpha=0$, and hence:

$$
r_{2}(0)=r_{2 B} \cdot\left(\frac{n-\cos \alpha_{B}}{n-1}\right)=\xi r_{o}+r_{o}+t r_{0}
$$

where $t$ represents the total lens thickness (see Figure 1).

\section{Lens Design and Simulation with UV-LED}

The goal of the present study is to design a collimating lens for UV-LEDs for use in proximity/contact UV photolithography applications, in which highly-collimated UV light is required. Once the UV light is collimated, a uniform distribution of the UV dosage over the wafer/substrate surface area can be achieved by overlapping the UV exposures of multiple LED/lens units arranged in the form of an array using a pre-programmed mechanical scanning movement.

In the method proposed in Section 2, only three parameters are required to complete the lens design, namely $r_{0}, \xi$, and $\theta_{B}$. However, since there is only one characteristic length $\left(r_{o}\right)$ involved in the analysis, $r_{O}$ can be set equal to one such that all of the other dimensions become normalized with respect to $r_{0}$. As a result, only two parameters, i.e., $\xi$ and $\theta_{B}$, were actually needed for the lens design under a point-source assumption. In real applications based on the use of true LEDs as the light source, the light is actually emitted from a finite size area with a dimension of $d$. The larger the ratio of $r_{o}$ to $d$, the better the point source assumption is, and hence the better the light collimating effect achieved by the proposed double freeform lens. Regarding the two design parameters, $\xi$ dominates the shape of the lens profile and the Fresnel losses, while $\theta_{B}$ determines the total light collection angle from the LED and therefore governs the energy efficiency of the collimated LED light.

The present study used an NCSU276A UV-LED (Nichia Inc., Tokushima, Japan) for illustration purposes. As shown in Table 1, the main features of the UV-LED include a peak wavelength of $365 \mathrm{~nm}$, an optical power of $780 \mathrm{~mW}$, and a radiation angle of $130^{\circ}$ (full angle at half intensity). The analysis was performed using commercial Zemax optical design software (OpticStudio, Kirkland, WA, USA). Figure 2a,b show the angular distribution of the illumination intensity of the NCSU276A UV-LED, as simulated using the source model file provided by Nichia Inc. [21]. The results confirmed that the full angle of divergence measured at half the maximum intensity was indeed around $130^{\circ}$. Furthermore, the light emitting area size is around $1 \times 1 \mathrm{~mm}^{2}$.

Table 1. Main features of NCSU276A UV-LED (Nichia Inc., Tokushima, Japan).

\begin{tabular}{cc}
\hline Model No. & NCSU276A \\
\hline Peak Spectrum & $365 \mathrm{~nm}$ \\
Typical Optical Power & $780 \mathrm{~mW}$ \\
Forward Voltage $\left(V_{F}\right)$ & $3.8 \mathrm{~V}$ (Typ.) \\
Directivity (2 $\theta$, Half Max.) & $4.4 \mathrm{~V}$ (Max.) \\
Forward Current $\left(I_{F}\right)$ & $130^{\circ}$ \\
LED Size & $500 \mathrm{~mm}$ \\
Light Emitting Area & $3.5 \times 3.5 \times 2.0 \mathrm{~mm}^{3}$ \\
\end{tabular}




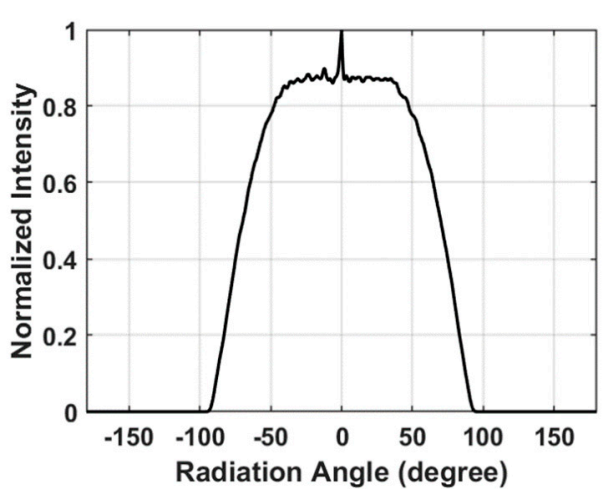

(a)

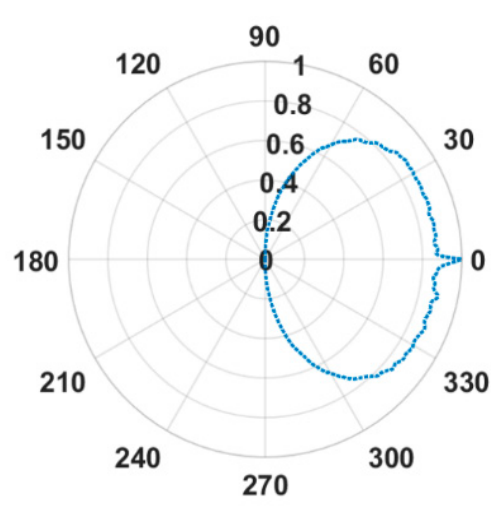

(b)

Figure 2. Angular distribution of radiation intensity of NCSU276A ultra violet light emitting diode (UV-LED): (a) Rectangular plot and (b) polar plot.

The UV-LED collimating lens designed in this study was manufactured using an injection molding technique since it can precisely control the lens surface profiles and enables the mass production of the lens with a high throughput and low cost. The lens was manufactured in PLEXIGLAS ${ }^{\circledR} 8012$ (Evonik Performance Materials $\mathrm{GmbH}$, Essen, Germany), which is a poly(methyl methacrylate) (PMMA) material suitable for making lenses with a working UV-A spectrum of 365 395 nm and has an optical refraction index $(n)$ of $1.51-@ 365 \mathrm{~nm}$ [22]. Figure 3a,b show the calculated optical transmission coefficients of the chosen material at the air/lens and lens/air interfaces, respectively, as a function of the incident angle (note that the calculated coefficients are used in the later lens design process). As shown in Figure 3b, the total reflection angle at the PMMA/air interface was around $41^{\circ}$.

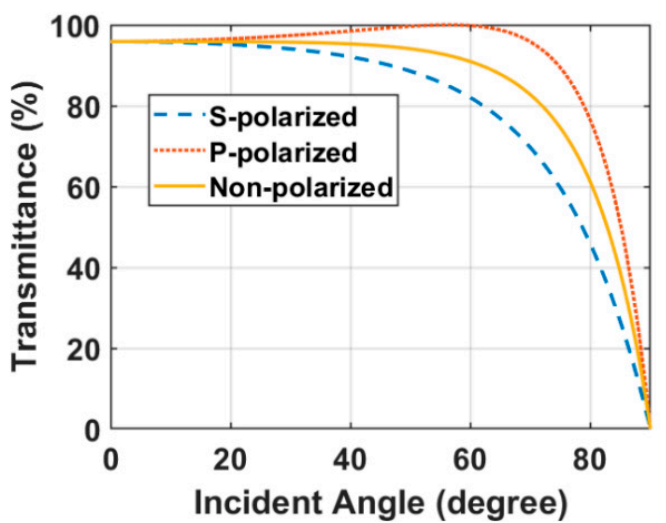

(a)

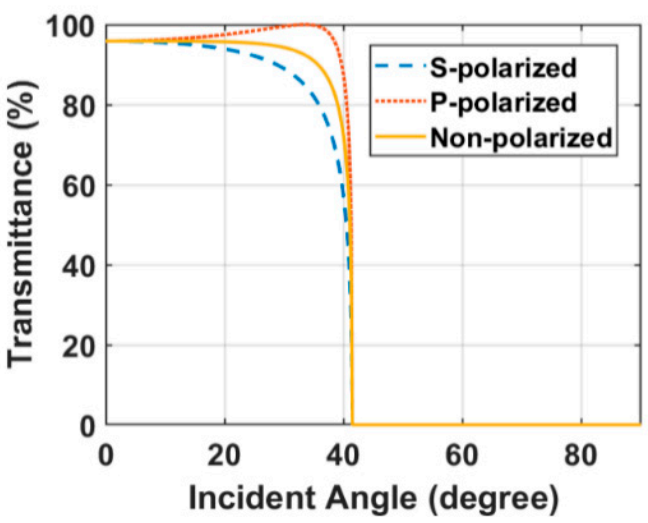

(b)

Figure 3. Fresnel optical transmittance as function of incident angle for: (a) Air to poly(methyl methacrylate) (PMMA) interface, and (b) PMMA to air interface. (Note that the refraction index of the chosen PMMA material is 1.51).

Using the method described in Section 2, several double freeform lenses were designed with $\xi$-parameter values ranging from 0.1 to 0.9 . The calculated $S_{1}$ and $S_{2}$ surfaces obtained from Equations $(6 a, b)$ and (12) are shown in Figure $4 a, b$, respectively (note that the refraction index $(n)$ was taken as 1.51 in every case and the collecting angle $\left(\theta_{B}\right)$ was set as $\left.60^{\circ}\right)$. The numerical labels in Figure $4 \mathrm{a}$,b represent the Fresnel transmittance values at the corresponding locations on the boundary surface. It is seen that for the $S_{1}$ surface, most of the optical transmittance values were around $95 \%$ for radiation angles $\theta=0^{\circ} \sim 60^{\circ}$ irrespective of the value of $\xi$. However, for the $S_{2}$ surface, the optical transmittance at higher radiation angles reduced rapidly (and even reached zero) with increasing $\xi$ 
due to the effects of internal total reflection. In other words, the flux energy received at larger radiation angles of the LED was completely rejected by the lens.

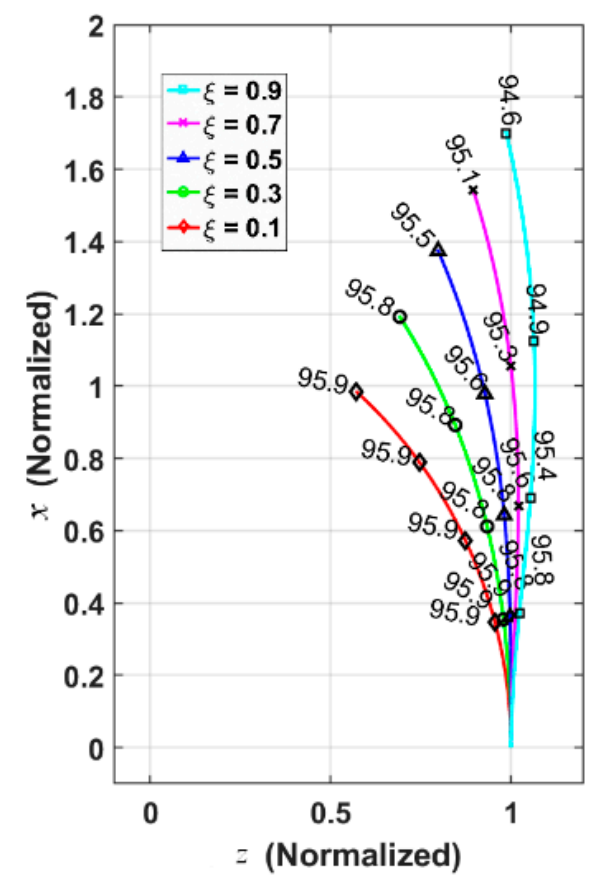

(a)

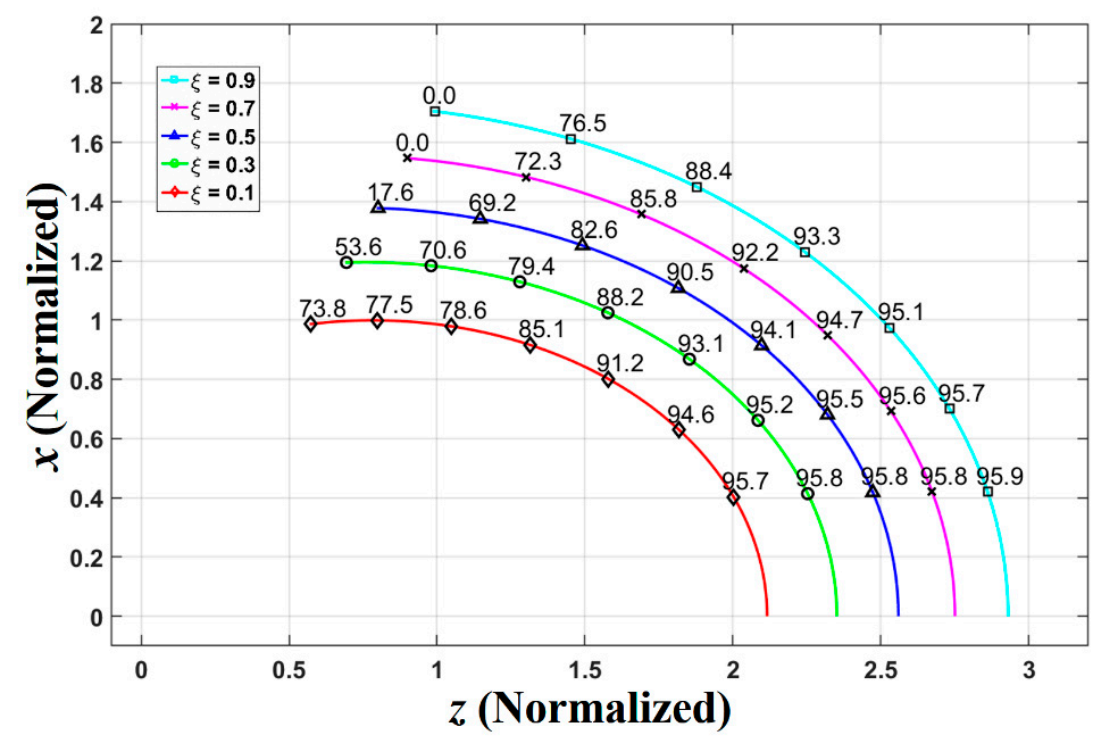

(b)

Figure 4. Designed lens surface profiles for different values of $\xi$ : (a) Surface $S_{1}$ and (b) surface $S_{2}$. (Note that the numerical labels show the Fresnel transmittance percentages at the corresponding locations).

Based on the angular distribution of the radiation intensity of the chosen NCSU276A UV-LED (see Figure 2a,b) and the Fresnel losses computed at surfaces $S_{1}$ and $S_{2}$ (see Figure $4 \mathrm{a}, \mathrm{b}$ ), one can roughly estimate the intensity distribution of the collimated LED beam on a projected plane perpendicular to the $z$-axis. Figure 5 presents the corresponding results for the $\xi$ values considered above. In the present study, the lens profile design calculated for $\xi=0.3$ was chosen for illustration purposes since, 
compared to larger values of $\xi$, a value of $\xi=3$ generally resulted in lower Fresnel losses and limits the lens aperture size and projected area size thereby assuring a higher average projected light intensity. Moreover, a smaller value of $\xi$ (e.g., $\xi=0.1$ ) resulted in a positive slope of surface $S_{2}$ at point $B$ and hence complicates the computer numerical control (CNC) machining of the steel mold and makes the injection molding of the lens almost impossible.

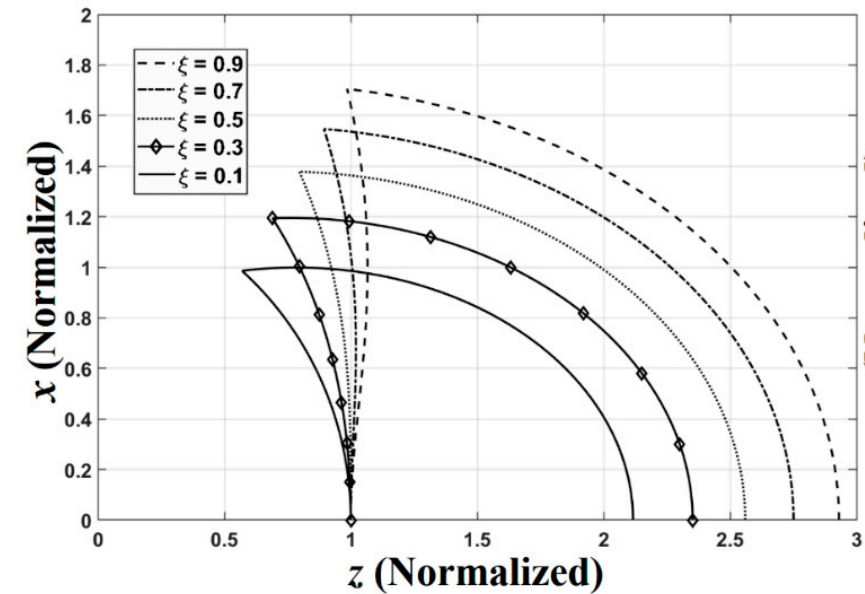

$z$ (Normalized)

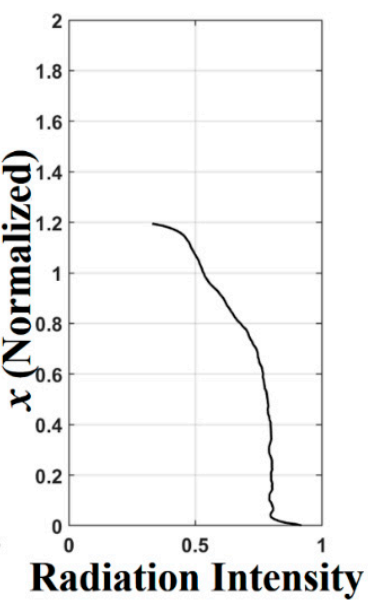

(Normalized)

Figure 5. Double freeform lens designs with different values of $\xi$ (left) and estimated intensity distribution of collimated beam on projected plane (right).

The optical performance of the double freeform lens with $\xi=0.3$ was evaluated by means of Zemax simulations. The simulations commenced by determining the collimating performance of the lens given the radiation pattern of the NCSU276A UV-LED shown in Figure 2. The simulation results showed that the light emerging from the lens was perfectly collimated with a zero divergent angle. In other words, the accuracy of the proposed collimating lens design under the assumption of a point source was confirmed.

The angular distribution of the collimated LED light was then re-simulated using the true finite-sized model of the NCSU276A LED provided by the manufacturer. In performing the simulations, the LED illuminating chip size was set as $1.0 \times 1.0 \mathrm{~mm}^{2}$, while the distance between the LED and lens surface (i.e., $r_{o}$ in Figure 1) was set as $10.65 \mathrm{~mm}$ based on a practical consideration of the injection-molded lens size and the LED intensity level after collimating. Based on the light source model of the NCSU276A, one million rays were used in the Zemax simulation process together with design parameters of $\xi=0.3, \theta_{B}=60^{\circ}$, and $r_{o}=10.65 \mathrm{~mm}$. The angular distribution of the light intensity was statistically analyzed at a detector plane with a size of $40 \times 40 \mathrm{~mm}^{2}$ located at $\mathrm{z}=+40 \mathrm{~mm}$. Figure 6a shows a two-dimensional view of the ray-tracing results between the finite LED light source, double freeform lens, and detector plane. As shown, not all of the rays collimated and incident on the detector plane. Thus, while the LED used in the simulations had a total emitting power of $821 \mathrm{~mW}$, the total light power received on the detector plane was just $400.3 \mathrm{~mW}$. In other words, the double freeform collimating lens had an energy efficiency of $48.8 \%$, where the losses were due mainly to the collecting angle of the lens and the Fresnel losses at the air/lens interfaces.

To better understand the light collimating effect of the double freeform lens, for each ray reaching the detector plane, the collimated angle $\gamma$, that is, the angle between the incident ray and the $+z$ axis, was determined, together with the associated light energy of the ray following refraction by lens surfaces $S_{1}$ and $S_{2}$. A statistical analysis was then performed to determine the variation of the total light intensity with the collimating angle $\gamma$. As shown in Figure $6 \mathrm{~b}$, around one half $(50 \%)$ of the collimated UV-LED light intensity was provided by rays with a collimating angle of less than $1.56^{\circ}$, while around $90 \%$ of the intensity was contributed by rays with a collimating angle of less than $3.62^{\circ}$. Notably, this 
performance was better than that of a conventional mercury lamp and fly's eye homogenizer system, in which the typical collimating angle is around $1.5^{\circ}$ at a $50 \%$ light intensity level. In other words, the proposed double freeform lens and UV-LED have good potential to replace the mercury light sources used in conventional lithography applications within the industry.

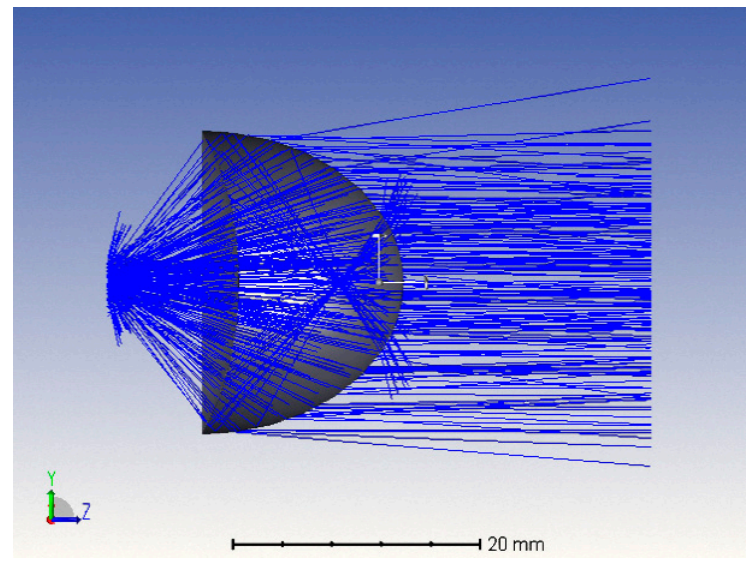

(a)

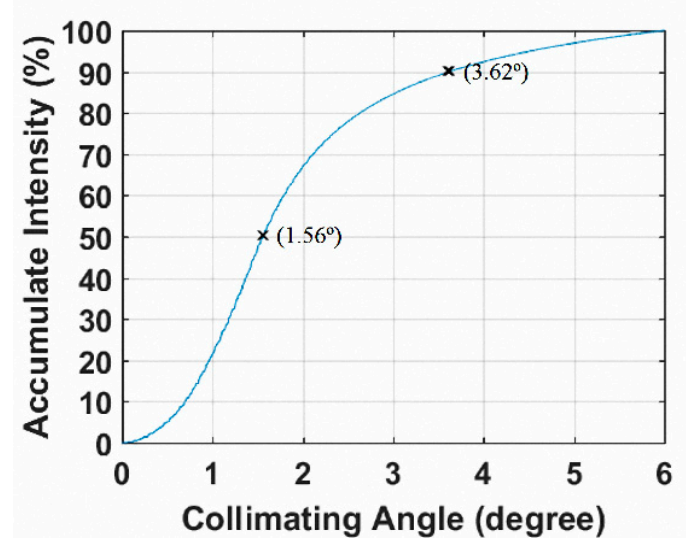

(b)

Figure 6. Zemax simulation results for NCSU276A UV-LED and double freeform lens $(\xi=0.3$, $\theta_{B}=60^{\circ}$ and $r_{o}=10.65 \mathrm{~mm}$ ): (a) $2 \mathrm{D}$ view of ray tracing results in Zemax, and (b) percentage of accumulated light intensity within collimating angle $(\gamma)$.

\section{Experiment Results}

As described in the previous section, the designed double freeform lens was manufactured by injection molding in UV-grade PMMA polymer PLEXIGLAS ${ }^{\circledR} 8012$ (Evonik Industries, Essen, Germany). A two-piece steel mold was first fabricated by a CNC machining system in accordance with the designed lens profile shown in Figure 5 for $\xi=0.3, \theta_{B}=60^{\circ}$, and $r_{o}=10.65 \mathrm{~mm}$. The dividing point between the profiles of the two injection molds was set as point $B$. Mass-production of the PMMA lens was then carried out using a standard injection molding system. Figure 7 shows a photograph of the double freeform lens produced in a typical molding cycle.

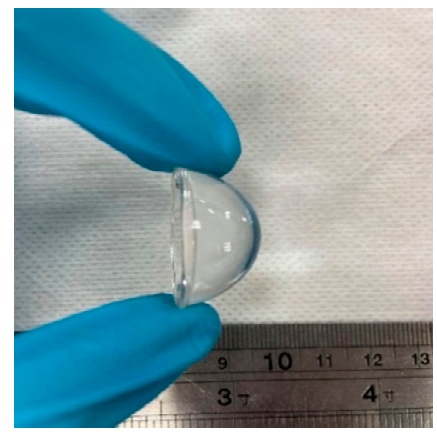

(a)

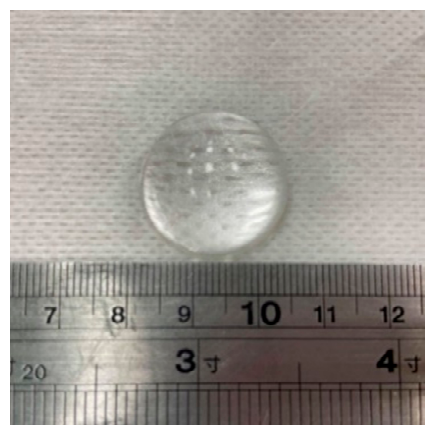

(b)

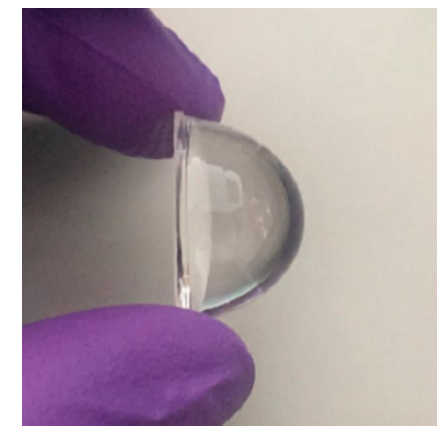

(c)

Figure 7. Photograph of double freeform lens produced by injection molding of UV-graded PMMA polymer. (a) Side view; (b) Front view; (c) Close side view

Figure 8 presents a schematic illustration of the experimental setup used to measure the optical performance of the fabricated lens with the NCSU276A UV-LED illumination source. As shown, the system consisted mainly of the collimating lens and UV-LED with correct distance and positioning between them, a CCD camera (FLIR Systems Inc., CM3-U3-13S2M-CS, Wilsonville, OR, USA), and a 
pin-hole plate. The pin-hole had a diameter of $50 \mu \mathrm{m}$ and was placed at a distance of $4 \mathrm{~mm}$ from the sensing pixels of the CCD. Moreover, the assembled pin-hole/CCD unit was mounted such that it faced the lens/LED along its optical axis (z-axis). Consequently, following the lens collimation, the LED light passed through the pin-hole and was incident on the sensing pixels of the CCD. The intensity and distribution of the light incident on the pixel surface were determined using a calibration process with an optical power meter. Briefly, the distribution of the light intensity with respect to the incident angle was obtained using a simple geometry relationship between each sensing pixel and the pin-hole, while the overall light intensity and its distribution as a function of the collimating angle was determined by scanning the pin-hole/CCD or lens/LED along both $x$ - and $y$-directions. The LED used in the experiment had a total emitting power of $348.5 \mathrm{~mW}$ under drive current of $500 \mathrm{~mA}$, and the total light power received on the detector plane was just $152.6 \mathrm{~mW}$. In other words, the double freeform collimating lens had an energy efficiency of $43.79 \%$. Figure $8 \mathrm{~b}$ shows the results obtained for the accumulated light intensity within a collimating angle of $\gamma$. It is seen that around one half $(50 \%)$ of the collimated UV-LED light intensity was obtained within a collimating angle of less than $1.88^{\circ}$, while $90 \%$ of the intensity was obtained within a collimating angle of less than $4.84^{\circ}$. In other words, the experimental results are in good qualitative agreement with the simulation results shown in Figure $6 \mathrm{~b}$. The discrepancy between the two sets of results arose most likely from machining errors of the lens profiles, experimental misalignments between the lens and the LED, and slight variations in the lens material constants. Nevertheless, the overall performance of the proposed lens in collimating the LED light was confirmed.

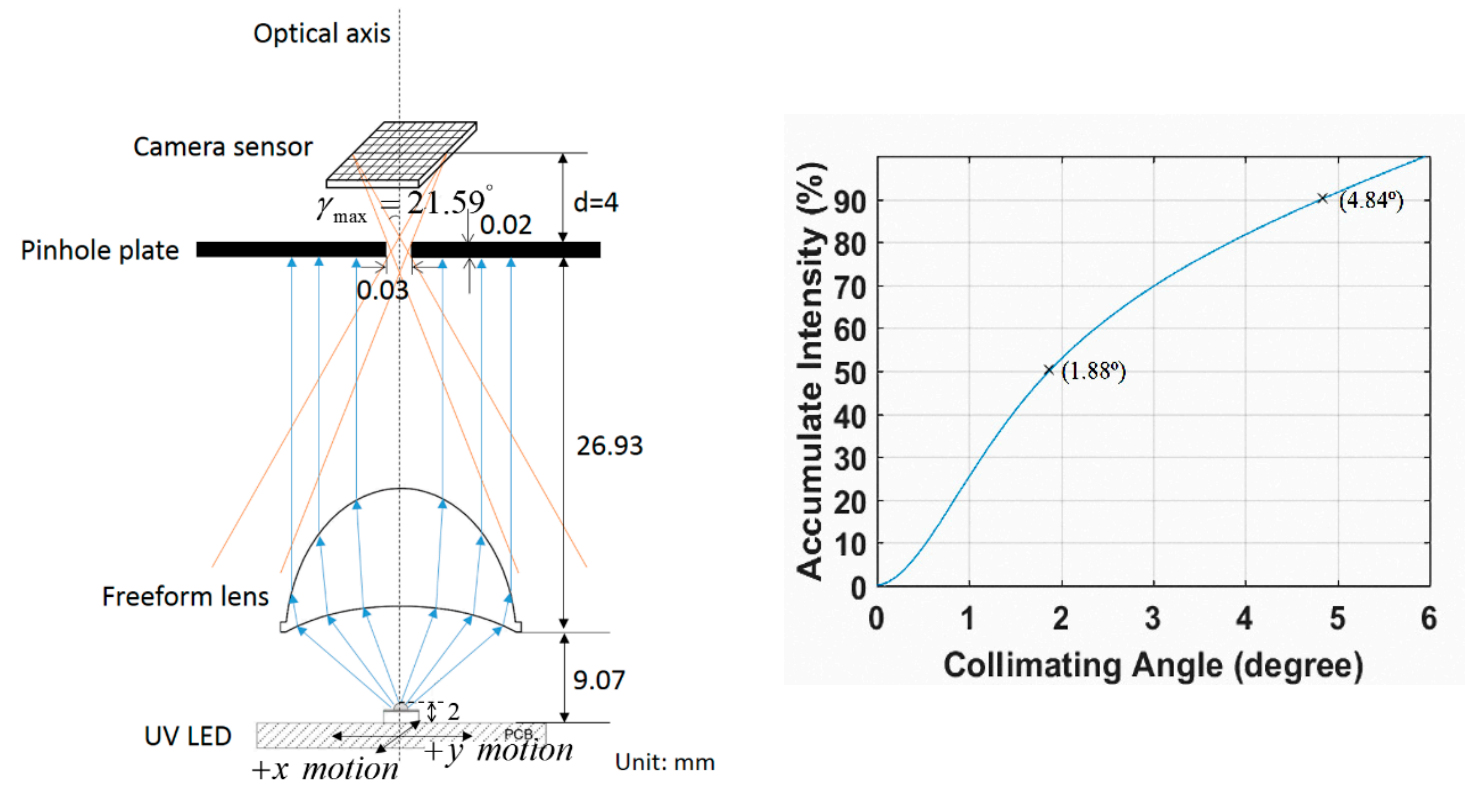

(a)

(b)

Figure 8. Experimental measurement results for double freeform collimating lens with NCSU276A UV-LED: (a) Schematic illustration of experimental setup, and (b) measured total light intensity within collimating angle $(\gamma)$.

When applying UV-LEDs to conventional proximity/contact photolithography, two of the most important requirements include a small collimating angle and a uniform distribution of the UV light intensity over a large area. The results presented in Figure 8 confirm the ability of the proposed double freeform lens to collimate the UV light from a UV-LED with a small collimating angle. In other words, the proposed lens satisfied the first requirement. However, to meet the second requirement, it is necessary to utilize an array of LED/lens units and an oblique scanning method. For example, 
as shown schematically in Figure 9a, a tilted array of LED/lens units were deployed at an angle with respect to the nominated scanning direction (e.g., the $y$-axis) and the arrayed UV light sources are then scanned together across the surface of the photoresist-coated substrate at a constant speed. Each LED/lens unit in the array draws its own scanning line, and the aim is thus to equally space all of the scanning lines such that the UV light energy is evenly distributed in both the $x$ - and the $y$-directions. As shown in Figure 9a, such an approach results in an effective exposure area in the central area of the array within which the accumulated UV light intensity is uniform and can hence be applied for photolithography. The proposed double freeform lens had an aperture diameter of $24.5 \mathrm{~mm}$. Hence, the center-to-center distance in the $6 \times 6$ array was chosen as $25.4 \mathrm{~mm}$ (see Figure 9a) such that the assembly of the arrayed LED/lens units was mechanically feasible. Figure $9 \mathrm{~b}$ shows the simulation results obtained for the accumulated UV intensity after scanning by the array (note that the results are based on the simulated radiation intensity pattern for each LED/lens unit given in Figure 5). The results confirm that a uniform intensity distribution was obtained within the effective exposure area. It is noted that in real applications, the intensity uniformity is inevitably degraded by the variation and positional accuracy of the UV-LEDs and lenses in the array. However, the simulation results presented in Figure $9 \mathrm{~b}$ nevertheless confirmed the general feasibility of applying LED/lens units for large-area photolithography.

(a)
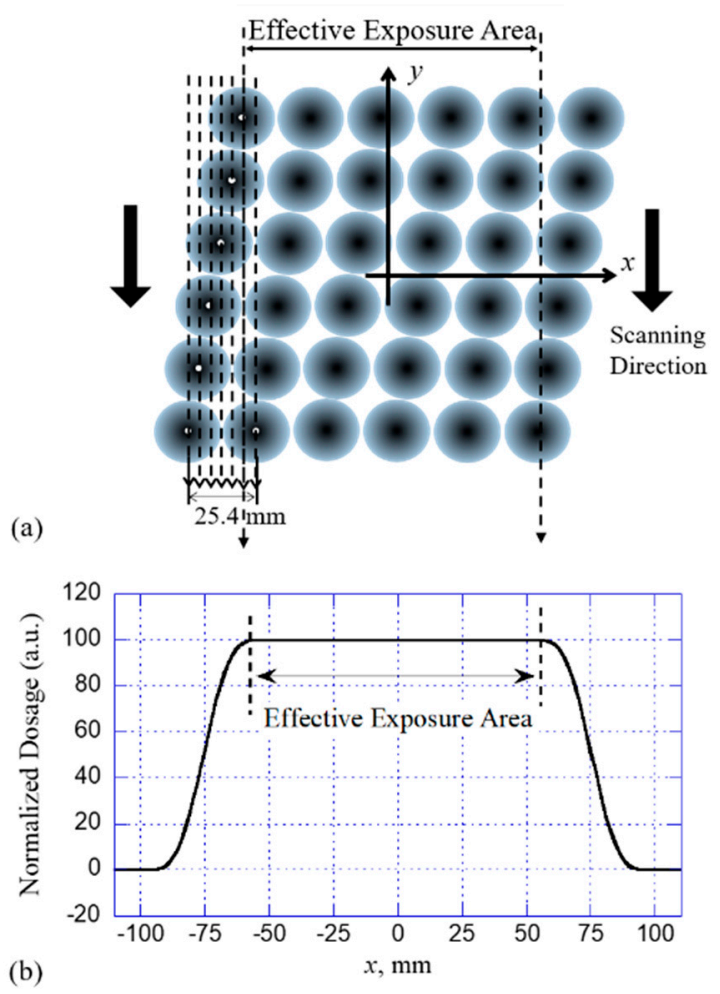

Figure 9. (a) Illustrative example of UV-LED/lens unit array for oblique photolithography scanning, and (b) simulated UV intensity distribution and its uniformity over effective exposure area.

\section{Conclusions}

This paper presented a double-sided freeform lens for collimating light emitted from a LED. In developing the proposed lens, the analyses assumed a point light source, and the continuous lens profiles were characterized mathematically by polar coordinates. It was shown that the two surface profiles of the lens could be analytically represented and determined using an ordinary differential equation and a closed-form solution, respectively. Notably, the overall performance of the lens/LED unit could be adjusted by tuning just two design parameters. As a result, the proposed double 
freeform lens provided a more attractive and feasible solution for collimating light produced by commercial UV-LEDs.

The optical performance of the proposed double freeform lens was simulated for a commercial UV-LED with a finite light emitting area of approximately $1 \times 1 \mathrm{~mm}^{2}$ and a full radiation angle of $130^{\circ}$. The simulation results showed that the lens had a power efficiency of approximately $48.8 \%$ and the power efficiency of experiment's result was $43.79 \%$. In addition, the collimating angles for $50 \%$ and $90 \%$ of the total light intensity were $1.56^{\circ}$ and $3.62^{\circ}$, respectively. A prototype lens was fabricated in UV-grade PMMA polymer using a standard injection molding technique. The optical performance of the lens/LED was characterized experimentally. It was shown that for a lens aperture diameter of $24.5 \mathrm{~mm}$ with a central distance of $10.65 \mathrm{~mm}$ between the lens and the LED, and an opening aperture angle of $\pm 60^{\circ}$, the collimating angles for $50 \%$ and $90 \%$ of the total light intensity were $1.88^{\circ}$ and $4.84^{\circ}$, respectively. The results therefore confirmed the potential for applying the proposed double freeform lens and UV-LED not only to photolithography, but also to other applications, such as a printed circuit board and integrated circuit package.

Author Contributions: Conceptualization, Y.-C.L.; Data curation, Y.-S.S.; Formal analysis, C.-Y.W.; Methodology, Y.-C.L.; Project administration, Y.-C.L.; Resources, C.-Y.W.; Software, Y.-S.S. and C.-Y.W.; Supervision, Y.-C.L.; Validation, Y.-S.S.; Visualization, C.-Y.W.; Writing-Original draft, Y.-S.S.

Funding: This research was funded by the Southern Taiwan Science Park Bureau and Ministry of Science and Technology of Taiwan under Project Nos. 106GF08, MOST 106-3114-8-006-003 and MOST 107-2823-8-006-004.

Acknowledgments: The authors are grateful for the technical and equipment support provided by the Center for Micro/Nano Science and Technology at National Cheng Kung University (Taiwan).

Conflicts of Interest: The authors declare no conflict of interest.

\section{References}

1. Ding, Y.; Liu, X.; Zheng, Z.-R.; Gu, P.-F. Freeform LED lens for uniform illumination. Opt. Express 2008, 16, 12958-12966. [CrossRef] [PubMed]

2. Luo, Y.; Feng, Z.; Han, Y.; Li, H. Design of compact and smooth free-form optical system with uniform. illuminance for LED source. Opt. Express 2010, 18, 9055-9063. [CrossRef] [PubMed]

3. Zhao, Z.; Zhang, H.; Zheng, H.; Liu, S. New reversing freeform lens design method for led uniform illumination with extended source and near field. Opt. Commun. 2018, 410, 123-129. [CrossRef]

4. Hu, R.; Gan, Z.; Luo, X.; Zheng, H.; Liu, S. Design of double freeform-surface lens for LED uniform illumination with minimum Fresnel losses. Optik 2013, 124, 3895-3897. [CrossRef]

5. Wu, H.; Zhang, X.; Ge, P. Double freeform surfaces lens design for LED uniform illumination with high distance-height ratio. Opt. Laser Technol. 2015, 73, 166-172. [CrossRef]

6. Wu, R.; Huang, C.Y.; Zhu, X.; Cheng, H.; Liang, R. Direct three-dimensional design of compact and ultraefficient freeform lenses for extended light sources. Optica 2016, 3, 840-843. [CrossRef]

7. Wang, K.; Chen, F.; Liu, Z.Y.; Luo, X.B.; Liu, S. Design of compact freeform lens for application specific. Light-Emitting Diode packaging. Opt. Express 2010, 18, 413-425. [CrossRef] [PubMed]

8. Wang, S.; Wang, K.; Chen, F.; Liu, S. Design of primary optics for LED chip array in road lighting. Application. Opt. Express 2011, 19 (Suppl. S4), A716-A724. [CrossRef]

9. Hu, R.; Luo, X.; Zheng, H.; Zong, Q.; Gan, Z.; Wu, B.; Liu, S. Design of a novel freeform lens for LED uniform illumination and conformal phosphor coating. Opt. Express 2012, 20, 13727-13737. [CrossRef] [PubMed]

10. Zhenrong, Z.; Xiang, H.; Xu, L. Freeform surface lens for LED uniform illumination. Appl. Opt. 2009, 48, 6627-6634. [CrossRef] [PubMed]

11. Zhao, S.; Wang, K.; Chen, F.; Wu, D.; Liu, S. Lens design of LED searchlight of high brightness and distant spot. J. Opt. Soc. Am. A 2011, 28, 815-820. [CrossRef] [PubMed]

12. Chen, J.; Wang, T.; Huang, K.; Liu, T.; Tsai, M.; Lin, C. Freeform lens design for LED collimating illumination. Opt. Express 2012, 20, 10984-10995. [CrossRef] [PubMed]

13. Tsai, C.-Y. Design and analysis of reflector for uniform light-emitting diode illuminance. J. Opt. Soc. Am. A 2013, 30, 993-1001. [CrossRef] [PubMed] 
14. Chen, Y.; Wu, S.W.Y.; Ren, Y.; Guan, W.; Zhou, Y. Long-range visible light communication system based on LED collimating lens. Opt. Commun. 2016, 377, 83-88. [CrossRef]

15. Pan, C.-T.; Yang, T.-L.; Chen, Y.-C.; Chang, C.-M.; Hsu, J.-H.; Huang, J.-C. Design of freeform lens with TFMG reflector for UV-LEDs lithography system. Optik 2016, 127, 6850-6857. [CrossRef]

16. Kuo, S.-H.; Chen, C.-F. Design of a collimated UV-LED exposure unit based on light spread function method. Appl. Opt. 2017, 56, 5542-5549. [CrossRef] [PubMed]

17. Luo, T.; Wang, G. Compact collimators designed with a modified point approximation for light emitting diodes. Proc. SPIE 2017, 10379, 103790G.

18. Hui, X.; Liu, J.; Wan, Y.; Lin, H. Realization of uniform and collimated light distribution in a single freeform-Fresnel double surface LED lens. Appl. Opt. 2017, 56, 4561-4565. [CrossRef] [PubMed]

19. Zhao, Z.; Zhang, H.; Liu, S.; Wang, X. Effective freeform TIR lens designed for LEDs with high angular color uniformity. Appl. Opt. 2018, 57, 4216-4221. [CrossRef] [PubMed]

20. N-BK7 Positive Meniscus Lenses, (Thorlabs, Inc., United States). Available online: https://www.thorlabs. com/newgrouppage9.cfm?objectgroup_id=130 (accessed on 23 October 2019).

21. Source Model File for Zemax Software Nichia Inc. NCSU276A UV-LED. Available online: https://www. nichia.co.jp/specification/products/led/NCSU276A-E.pdf (accessed on 23 October 2019).

22. PLEXIGLAS®8012 Material Manual, (Evonik Performance Materials GmbH, Essen, Germany). Available online: https://corporate.evonik.com/en/ (accessed on 23 October 2019).

(C) 2019 by the authors. Licensee MDPI, Basel, Switzerland. This article is an open access article distributed under the terms and conditions of the Creative Commons Attribution (CC BY) license (http://creativecommons.org/licenses/by/4.0/). 